Y. G. LU

Nagoya Math. J.

Vol. 145 (1997), 1-28

\title{
ON THE INTERACTING FREE FOCK SPACE AND THE DEFORMED WIGNER LAW
}

\author{
Y. G. LU
}

\section{\$1. Introduction}

The Fock space is a basic structure for the quantum field theory and quantum stochastic calculus. In all the cases, a Fock space can be described as a direct sum of a sequence of some Hilbert spaces, i.e. a Fock space has the form of $\bigoplus_{n=0}^{\infty} \mathscr{H}_{n}$,where, $\mathscr{H}_{0}:=\mathbf{C}$ is the complex field and $\mathscr{H}_{1}:=\mathscr{H}$ is a given Hilbert space. Moreover,

i) the Boson Fock space corresponds to the case of $\mathscr{H}_{n}:=\mathscr{H}^{\circ n}$, where $\mathscr{H}^{\circ n}$ is the $n$-folds symmetric tensor product of $\mathscr{H}$;

ii) the Fermion Fock space corresponds to the case of $\mathscr{H}_{n}:=\mathscr{H}^{\wedge n}$, where $\mathscr{H}^{\wedge n}$ is the $n$-folds anti-symmetric tensor product of $\mathscr{H}$;

iii) the Free (or Full) Fock space corresponds to the case of $\mathscr{H}_{n}:=\mathscr{H}^{\otimes n}$ : the usual $n$-folds tensor product of $\mathscr{H}$.

In the consideration of the central limit of the time evolution operator of the quantum electric-magnetic field (see [1,2]), a new type of Free Fock structure is erquired in order to describe the limit of the time evolution operator. The limit is a quantum stochastic process satisfying a certain quantum stochastic differential equation.

In the new Free Fock structure, the $n$-th space $\mathscr{H}_{n}$ is not exactly equal to the $n$-folds tensor product Hibert space $\mathscr{H}^{\otimes n}$ : one obtains the $\mathscr{H}_{n}$ by introducing a scalar product $\langle\cdot, \cdot\rangle_{n}$ on the algebraic tensor product $\mathscr{H}^{\odot n}$ and for $f_{1}, g_{1}, \ldots, f_{n}$, $g_{n} \in \mathscr{H}$, in general, the product $\Pi_{h=1}^{n}\left\langle f_{k}, g_{k}\right\rangle$ is not the same as the scalar product $\left\langle f_{1} \odot \cdots \odot f_{n}, g_{1} \odot \cdots \odot g_{n}\right\rangle$, where, we have omitted the sub-index $n$ of $n$-th scalar product and throughout the paper, the same omission will be adopted.

Thus, the Free Fock (one could also make the same consideration for the Boson and the Fermion cases, see [3]) space has the form

Received April 10, 1995. 


$$
\Gamma_{0}(\mathscr{H})=\mathbf{C} \oplus \bigoplus_{n=1}^{\infty} \mathscr{H}_{n}
$$

and the $n$-th Hilbert space is $\left(\mathscr{H}^{\odot n},\langle\cdot, \cdot\rangle\right)$.

The present article is devoted to discuss such new Free Fock structure. Moreover, we shall restrict ourselves to the case in which

i) $\mathscr{H}:=L^{2}(M, d \mu)$, where, $M$ is a measurable space and $\mu$ is a $\sigma$-finite measure on $M$;

ii) there exists a sequence of functions $\left\{\lambda_{n}\left(x_{1}, x_{2}, \ldots, x_{n}\right)\right\}_{n=1}^{\infty}\left(\lambda_{n}: M^{n} \rightarrow\right.$ $\mathbf{R}_{+}$) and two sequences of positive numbers $\left\{b_{n}, d_{n}\right\}_{n=1}^{\infty}$, such that, for any $n \in \mathbf{N}$,

$$
0<\lambda_{n} \leq b_{n}, \quad \frac{\lambda_{n+1}\left(x_{1}, x_{2}, \ldots, x_{n}, x_{n+1}\right)}{\lambda_{1}\left(x_{1}\right) \cdot \lambda_{n}\left(x_{2}, \ldots, x_{n}, x_{n+1}\right)} \leq d_{n}
$$

iii) the scalar product of $n$-th Hilbert space is defined as:

$$
\begin{array}{r}
\left\langle f_{1} \odot \cdots \odot f_{n}, g_{1} \odot \cdots \odot g_{n}\right\rangle:=\int \mu\left(d x_{1}\right) \cdots \mu\left(d x_{n}\right) \lambda_{n}\left(x_{1}, \ldots, x_{n}\right) \\
\prod_{h=1}^{n}\left(\bar{f}_{h} g_{h}\right)\left(x_{h}\right)
\end{array}
$$

for any $f_{1}, g_{1}, \ldots, f_{n}, g_{n} \in \mathscr{H}$.

Such type of Fock space will be called the interacting Free Fock space over the Hilbert space $\mathscr{H}=L^{2}(M, d \mu)$ with the interacting functions $\left\{\lambda_{n}\right\}_{n=1}^{\infty}$. It is obvious that with the choice $\lambda_{n}=1(n=1,2, \ldots)$, one gets the usual Free Fock space over $\mathscr{H}$.

In the section 2 , we introduce some basic concepts like the creation, annihilation operators; calculate the action of any annihilation operator on each $n$-th space $\mathscr{H}_{n}$ (it is not so trivial like in the usual Free Fock space case) and formulate the joint distribution of any product of some creation and annihilation operators. The section 3 is devoted to introduce the deformed Gaussianity on the interacting Free Fock space. Finally, in the section 4, we study some examples; introduce what so called the deformed Wigner distribution and obtain the explicit expression of the density function of the deformed Wigner ditribution.

\section{§2. Creation and annihilation operators, the joint distributions}

Having defined the interacting Free Fock space, we shall, in this section, introduce the creation and annihilation operators and obtain the joint distribution of the operators. Such investigations is essential for setting quantum stochastic calculus theory on the interacting Free Fock space (see [4]). 
Definition (2.1). The vector

$$
\Phi:=1 \oplus 0 \oplus 0 \oplus \cdots
$$

is called the vacuum of the interacting Free Fock space $\Gamma_{0}(\mathscr{H})$. The operator $A^{+}(g)$, where $g \in \mathscr{H}$, defined by, for any $n \in \mathbf{N}$ and $g_{1}, \ldots, g_{n} \in \mathscr{H}$,

$$
A^{+}(g)\left[g_{1} \odot \cdots \odot g_{n}\right]:=g \odot g_{1} \odot \cdots \odot g_{n}
$$

is called the creation operator (with respect to $g \in \mathscr{H}$ ).

Lemma (2.2). For any $g \in \mathscr{H}$, the creation operator $A^{+}(g)$ maps $\mathscr{H}_{n}$ into $\mathscr{H}_{n+1}$ and is bounded on each $\mathscr{H}_{n}$ :

$$
\left\|A^{+}(g)\right\| \leq \sqrt{d_{n}} \cdot\|g\|
$$

Proof. By the definition

$$
\begin{aligned}
& \left\|A^{+}(g)\left[g_{1} \odot \cdots \odot g_{n}\right]\right\|^{2} \\
= & \left\|g \odot g_{1} \odot \cdots \odot g_{n}\right\|^{2} \\
= & \left\langle g \odot g_{1} \odot \cdots \odot g_{n}, g \odot g_{1}, \odot \cdots \odot g_{n}\right\rangle \\
= & \int \mu(d x) \mu\left(d x_{1}\right) \cdots \mu\left(d x_{n}\right) \lambda_{n+1}\left(x, x_{1}, \ldots, x_{n}\right)|g(x)|^{2} \cdot \prod_{n=1}^{n}\left|g_{h}\right|^{2}\left(x_{n}\right) \\
= & \int \mu\left(d x_{1}\right) \cdots \mu\left(d x_{n}\right) \lambda_{n}\left(x_{1}, \ldots, x_{n}\right) \prod_{n=1}^{n}\left|g_{h}\right|^{2}\left(x_{h}\right) \cdot \\
& {\left[\int \mu(d x) \lambda_{1}(x)|g(x)|^{2} \cdot \frac{\lambda_{n+1}\left(x, x_{1}, \ldots, x_{n}\right)}{\lambda_{1}(x) \lambda_{n}\left(x_{1}, \ldots, x_{n}\right)}\right] . }
\end{aligned}
$$

By our assumption on the interacting functions, the quantity [...] in the right hand side of (2.3) is less than or equal to $d_{n} \cdot\|g\|^{2}$. Thus we have obtained the proof.

Of course, $A^{+}(g)$ is not necessarily to be bounded on the interacting Free Fock space $\Gamma_{0}(\mathscr{H})$ and the boundness depends on the sequence of the interacting functions $\left\{\lambda_{n}\right\}_{n=1}^{\infty}$. But we have

\section{Corollary.}

i) $A^{+}(g)$ is, for any $g \in \mathscr{H}$, an operator densely defined on $\Gamma_{0}(\mathscr{H})$ and the set

$$
\Gamma_{0}:=\left\{\sum_{n=0}^{N} c_{n} G_{n}: N \in \mathbf{N}, G_{n} \in \mathscr{H}_{n}, c_{n} \in \mathbf{C}, n=0,1,2, \cdots\right\}
$$


is included in $\mathscr{D}\left(A^{+}(g)\right)$ : the domain of $A^{+}(g)$.

ii) If $\sup _{n} \sqrt{d}_{n}<\infty$, then for any $g \in \mathscr{H}$, the creation operator $A^{+}(g)$ is bounded on $\Gamma_{0}(\mathscr{H})$ and

$$
\left\|A^{+}(g)\right\| \leq \sup _{n} \sqrt{d}_{n} \cdot\|g\|
$$

In the usual Free Fock space, $b_{n}=d_{n}=1$, so any creation operator is bounded.

Since $A^{+}(g)$ is an operator densely defined, its essential adjoint exists and will be denoted by $A(g)$, named by the annihilation operator (with respect to $g \in$ $\mathscr{H})$.

Lemma (2.3). For any $g \in \mathscr{H}$, the annihilation operator $A(g)$ possesses the following properties:

$$
\begin{gathered}
A(g) \mathscr{H}_{0}=0, \quad A(g): \mathscr{H}_{n} \rightarrow \mathscr{H}_{n-1} \\
\Gamma_{0} \subset \mathscr{D}(A(g))
\end{gathered}
$$

and moreover, for any $n=1,2, \ldots, m \in \mathbf{N}, f_{2}, f_{3}, \ldots, f_{m} \in \mathscr{H}_{n}, G_{n} \in \mathscr{H}_{n}$,

$$
\left\langle f_{2} \odot \cdots \odot f_{m}, A(g) G_{n}\right\rangle=\delta_{n, m}\left\langle g \odot f_{2} \odot \cdots \odot f_{n}, G_{n}\right\rangle
$$

Proof. For any $n=1,2, \ldots, m \in \mathbf{N}, f_{2}, f_{3}, \ldots, f_{m}, G_{n} \in \mathscr{H}_{n}$, by the definition

$$
\left\langle f_{2} \odot \cdots \odot f_{m}, A(g) G_{n}\right\rangle=\left\langle A^{+}(g)\left[f_{2} \odot \cdots \odot f_{m}\right], G_{n}\right\rangle=\left\langle g \odot f_{2} \odot \cdots \odot f_{m}, G_{n}\right\rangle
$$

This shows that $\Gamma_{0} \subset \mathscr{D}(A(g))$; the left hand side of the $(2.4 \mathrm{c})$ is equal to zero if $m \neq n$. Moreover, in the case of $n=m$, the above scalar product is equal to the right hand side of the $(2.4 \mathrm{c})$.

In particular, the formula $(2.4 \mathrm{c})$ implies that, if $n=0$,

$$
\langle F, A(g) \Phi\rangle=0, \quad \forall F \in \Gamma_{0}
$$

and $A(g) \mathscr{H}_{n} \subset \mathscr{H}_{n-1}$. Therefore, the proof the thesis is compeleted.

Now, an interesting problem is to calculate the expression

$$
A(g)\left[g_{1} \odot \cdots \odot g_{n}\right]
$$

for $n \geq 1$. By the definitions of the creation and annihilation operators, one knows that for any $n=1,2, \ldots, \mathbf{N}, f_{1}, g_{1}, \ldots, f_{n}, g_{n} \in \mathscr{H}$, 


$$
\begin{aligned}
& \left\langle f_{2} \odot \cdots \odot f_{n}, A\left(f_{1}\right)\left[g_{1} \odot \cdots \odot g_{n}\right]\right\rangle \\
= & \left\langle f_{1} \odot f_{2} \odot \cdots \odot f_{n}, g_{1} \odot \cdots \odot g_{n}\right\rangle \\
= & \int \mu\left(d x_{1}\right) \cdots \mu\left(d x_{n}\right) \lambda_{n}\left(x_{1}, \ldots, x_{n}\right) \prod_{n=1}^{n}\left(\bar{f}_{h} \cdot g_{h}\right)\left(x_{h}\right) \\
= & \int \mu\left(d x_{2}\right) \cdots \mu\left(d x_{n}\right) \lambda_{n-1}\left(x_{2}, \ldots, x_{n}\right) \cdot \prod_{h=2}^{n}\left(\bar{f}_{h} \cdot g_{h}\right)\left(x_{h}\right) \\
& {\left[\int \mu\left(d x_{1}\right) \frac{\lambda_{n}\left(x_{1}, \ldots, x_{n}\right)}{\lambda_{n-1}\left(x_{2}, \ldots, x_{n}\right)}\left(\bar{f}_{1} \cdot g_{1}\right)\left(x_{1}\right)\right] }
\end{aligned}
$$

Thus, in fact we have proved that

Lemma (2.4). For any $n \in \mathbf{N}, G_{n} \in \mathscr{H}_{n}$ and $f, g \in \mathscr{H}$,

$$
\begin{gathered}
{\left[A(f)\left(g \odot G_{n}\right)\right]\left(x_{1}, \ldots, x_{n}\right)=G_{n}\left(x_{1}, \ldots, x_{n}\right) \cdot} \\
\int \mu(d x) \frac{\lambda_{n+1}\left(x, x_{1}, \ldots, x_{n}\right)}{\lambda_{n}\left(x_{1}, \ldots, x_{n}\right)}(\bar{f} \cdot g)(x)
\end{gathered}
$$

where, here and in the following, the 0 -th interactiong function $\lambda_{0}$ is defined as 1 .

The boundness of the annihilation operator is the same as that of the creation operators.

Lemma (2.5). For any $n \in \mathbf{N}$ and $f \in \mathscr{H}$, the annihilation operator $A(f)$ on the $n+1-$ space $\mathscr{H}_{n+1}$ is bounded and with the bound $\sqrt{d}_{n+1} \cdot\|f\|$.

Proof. The proof is the same as that of Lemma (2.2).

In the following, we consider the creation and annihilation operators only on $\Gamma_{0}$ (i.e. we do not distinguish the operators themselves with their restriction on $\Gamma_{0}$ ) and therefore, they are adjoint each other.

Now, we try to understand the vacuum expectation of a product of many creation and annihilation operators, i.e. to get more information on

$$
\left\langle\Phi, A^{\varepsilon(1)}\left(f_{1}\right) \cdots A^{\varepsilon(n)}\left(f_{n}\right) \Phi\right\rangle
$$

where, $n \in \mathbf{N}, \varepsilon \in\{0,1\}^{n}, f_{1}, \ldots, f_{n} \in \mathscr{H}$ and for any $\epsilon \in\{0,1\}$,

$$
A^{\epsilon}:= \begin{cases}A, & \text { if } \epsilon=0 ; \\ A^{+}, & \text {if } \epsilon=1\end{cases}
$$

Since $A(f) \mathscr{H}_{0}=0$, we get immediately that 
Lemma (2.6). The quantity (2.8) differs from zero only if $\varepsilon(n)=1, \varepsilon(1)=0$.

For any fixed $\varepsilon \in\{0,1\}^{n}$ with the property of $\varepsilon(n)=1$ and $\varepsilon(1)=0$, there is a unique $1 \leq m \leq \frac{n}{2}$ and $1 \leq k_{1}<k_{2}<\cdots<k_{2 m-1}<n$, such that

$$
\begin{gathered}
0=\varepsilon\left(k_{2 j}+1\right)=\varepsilon\left(k_{2 j}+2\right)=\cdots=\varepsilon\left(k_{2 j+1}\right) \\
1=\varepsilon\left(k_{2 j+1}+1\right)=\varepsilon\left(k_{2 j+1}+2\right)=\cdots=\varepsilon\left(k_{2 j+2}\right)
\end{gathered}
$$

for each $j=0,1, \ldots, m-1$. Where, we have introduced the conveniences $k_{2 m}:=$ $n$ and $k_{0}:=0$.

With the above notations, we are able to rewrite (2.8) as

$$
\left\langle\Phi, \prod_{h=k_{0}+1}^{k_{1}} A\left(g_{h}\right) \cdot \prod_{h=k_{1}+1}^{k_{2}} A^{+}\left(g_{h}\right) \cdots \prod_{h=k_{2 m-2}+1}^{k_{2 m-1}} A\left(g_{h}\right) \cdot \prod_{h=k_{2 m-1}+1}^{k_{2 m}} A^{+}\left(g_{h}\right) \Phi\right\rangle
$$

where and hereinafter, for any elements $a_{1}, \ldots, a_{r}$ in a certain group $G$, by $\Pi_{h=1}^{r} a_{h}$, we denote the ordered product $a_{1} \cdots a_{r}$.

A generalization of Lemma (2.4) can be stated as following:

Lemma (2.7). For any $m, n \in \mathbf{N}, f_{1}, \ldots, f_{m}, g_{1}, \ldots, g_{n} \in \mathscr{H}$, the expression

$$
\begin{gathered}
A\left(f_{m}\right) \cdots A\left(f_{1}\right)\left[g_{1} \odot \cdots \odot g_{n}\right] \\
:=A\left(f_{m}\right)\left[\cdots\left[A\left(f_{2}\right)\left[A\left(f_{1}\right)\left[g_{1} \odot \cdots \odot g_{n}\right]\right]\right] \cdots\right]
\end{gathered}
$$

is equal to zero if $m>n$; equal to

$$
\left\langle f_{1} \odot \cdots \odot f_{n}, g_{1} \odot \cdots \odot g_{n}\right\rangle
$$

if $m=n$ and equal to

(2.13) $\left[\left(g_{m+1} \odot \cdots \odot g_{n}\right) \cdot \Lambda_{n-m}\left(f_{m}, \ldots, f_{1} ; g_{1}, \ldots, g_{n}\right)\right]\left(x_{m+1}, \ldots, x_{n}\right)$

in the case of $m<n$ (which belongs to $\mathscr{H}_{n-m}$ ), where

$$
\begin{gathered}
\Lambda_{n-m}\left(f_{m}, \ldots, f_{1} ; g_{1}, \ldots, g_{n}\right)\left(x_{m+1}, \ldots, x_{n}\right) \\
:=\int \mu\left(d x_{1}\right) \cdots \mu\left(d x_{m}\right) \frac{\lambda_{n}\left(x_{1}, \ldots, x_{n}\right)}{\lambda_{n-m}\left(x_{m+1, \ldots,} x_{n}\right)} \prod_{h=1}^{m}\left(\bar{f}_{h} \cdot g_{h}\right)\left(x_{h}\right)
\end{gathered}
$$

Proof. Because of (2.12) and the fact:

$$
\Lambda_{n-n}\left(f_{1}, g_{1}, \ldots, f_{n}, g_{n}\right)=\left\langle f_{1} \odot \cdots \odot f_{n}, g_{1} \odot \cdots \odot g_{n}\right\rangle
$$

we know that the conclusions of Lemma (2.7) in the cases of $m>n$ and $m=n$ 
are particular cases of that in the case of $m<n$. In order to prove the last conclusion of Lemma (2.7), we should calculate the right hand side of (2.14) for $m<n$.

By the definition of annihilation operator, we know that $A\left(f_{1}\right)\left(g_{1} \odot \cdots \odot\right.$ $\left.g_{n}\right) \in \mathscr{H}_{n-1}$ is given by

$$
\begin{gathered}
{\left[A\left(f_{1}\right)\left(g_{1} \odot \cdots \odot g_{n}\right)\right]\left(x_{2}, \ldots, x_{n}\right)} \\
=\left(g_{2} \odot \cdots \odot g_{n}\right)\left(x_{2}, \ldots, x_{n}\right) \int \mu\left(d x_{1}\right) \frac{\lambda_{n}\left(x_{1}, \ldots, x_{n}\right)}{\lambda_{n-1}\left(x_{2}, \ldots, x_{n}\right)}\left(\bar{f}_{1} \cdot g_{1}\right)\left(x_{1}\right) .
\end{gathered}
$$

Now by acting the annihilator $A\left(f_{2}\right)$ on the two sides of (2.15), we find that

$$
\begin{aligned}
& {\left[A\left(f_{2}\right)\left[A\left(f_{1}\right)\left(g_{1} \odot \ldots \odot g_{n}\right)\right]\right]\left(x_{3}, \ldots, x_{n}\right) } \\
= & \left(g_{3} \odot \cdots \odot g_{n}\right)\left(x_{3}, \ldots, x_{n}\right) . \\
& \int \mu\left(d x_{2}\right) \frac{\lambda_{n-1}\left(x_{2}, \ldots, x_{n}\right)}{\lambda_{n-2}\left(x_{3}, \ldots, x_{n}\right)}\left(\bar{f}_{2} \cdot g_{2}\right)\left(x_{2}\right) \cdot \\
& \int \mu\left(d x_{1}\right) \frac{\lambda_{n}\left(x_{1}, \ldots, x_{n}\right)}{\lambda_{n-1}\left(x_{2}, \ldots, x_{n}\right)}\left(\bar{f}_{1} \cdot g_{1}\right)\left(x_{1}\right)
\end{aligned}
$$

and which is equal to

$$
\begin{aligned}
& \left(g_{3} \odot \cdots \odot g_{n}\right)\left(x_{3}, \ldots, x_{n}\right) \cdot \\
& \quad \int \mu\left(d x_{1}\right) \mu\left(d x_{2}\right) \frac{\lambda_{n}\left(x_{1}, \ldots, x_{n}\right)}{\lambda_{n-2}\left(x_{3}, \ldots, x_{n}\right)}\left(\bar{f}_{1} \cdot g_{1}\right)\left(x_{1}\right)\left(\bar{f}_{2} \cdot g_{2}\right)\left(x_{2}\right) \\
& \quad=\left[\left(g_{3} \odot \cdots \odot g_{n}\right) \cdot \Lambda_{n-2}\left(f_{2}, f_{1} ; g_{1}, g_{2}\right)\right]\left(x_{3}, \ldots, x_{n}\right) .
\end{aligned}
$$

By repeating the above arguments and applying the induction, we finish the proof.

A trivial generalization of Lemma (2.7) is that

Lemma (2.8). For any $m, n \in \mathbf{N}, f_{1}, \ldots, f_{m} \in \mathscr{H}_{\text {and }} G_{n} \in \mathscr{H}_{n}$,

$$
A\left(f_{m}\right) \cdots A\left(f_{1}\right) G_{n}=V_{n-m}\left(f_{m}, \ldots, f_{1} ; G_{n}\right)
$$

where, $V_{n-m}\left(f_{m}, \ldots, f_{1} ; G_{n}\right)$ is defined as zero if $m>n$;

$$
\left\langle f_{1} \odot \cdots \odot f_{n}, G_{n}\right\rangle
$$

if $m=n$ and $i n$ the case of $m<n, V_{n-m}\left(f_{m}, \ldots, f_{1} ; G_{n}\right) \in \mathscr{H}_{n-m}$ is defined as

$$
\begin{aligned}
& V_{n-m}\left(f_{m}, \ldots, f_{1} ; G_{n}\right)\left(x_{m+1}, \ldots, x_{n}\right) \\
:= & \int \mu\left(d x_{1}\right) \cdots \mu\left(d x_{m}\right) \frac{\lambda_{n}\left(x_{1}, \ldots, x_{n}\right)}{\lambda_{n-m}\left(x_{m+1}, \ldots, x_{n}\right)} \prod_{h=1}^{m} \bar{f}_{h}\left(x_{h}\right) \cdot G_{n}\left(x_{1}, \ldots, x_{n}\right)
\end{aligned}
$$


Lemma (2.9). The quantity (2.11) differs from zero only if i) for any $j=0,1, \ldots, m-1$,

$$
\begin{aligned}
& \left(k_{2 j+1}-k_{2 j}\right)+\left(k_{2 j+3}-k_{2 j+2}\right)+\cdots+\left(k_{2(m-1)+1}-k_{2(m-1)}\right) \\
\leq & \left(k_{2(j+1)}-k_{2 j+1}\right)+\left(k_{2(j+2)}-k_{2 j+3}\right)+\cdots+\left(k_{2 m}-k_{2(m-1)+1}\right)
\end{aligned}
$$

and

ii) (2.20) takes equality for $j=0$, i.e.

$$
2\left(k_{1}+k_{3}+\cdots+k_{2(m-1)+1}\right)=2\left(k_{2}+k_{4}+\cdots+k_{2(m-1)}\right)+k_{2 m}
$$

'Proof. By Lemma (2.7) and Lemma (2.8), for any $j=0,1,2, \ldots, m-1$, if (2.20) is not true, then in a certain step we shall have an annihilation operator to act on $\mathscr{H}_{0}$ and which gives zero.

Moreover, if the left hand side of (2.21) is strictly less than the right hand side, then the vector

$$
\prod_{h=k_{0}+1}^{k_{1}} A\left(g_{h}\right) \cdot \prod_{h=k_{1}+1}^{k_{2}} A^{+}\left(g_{h}\right) \cdots \prod_{h=k_{2 m-2}+1}^{k_{2 m-1}} A\left(g_{h}\right) \cdot \prod_{h=k_{2 m-1}+1}^{k_{2 m}} A^{+}\left(g_{h}\right) \Phi
$$

belongs to $\mathscr{H}_{p}$ for some $p \geq 1$. Therefore, in this case, (2.11) must be equal to zero.

Remark. It follows easily from Lemma (2.9) that (2.11) (or equivalently, (2.8)) differs from zero only if $n$ is even. So, we are able to assume that $n=2 N$ for some $N \in \mathbf{N}$.

Now, we are ready to calculate (2.11) for $1 \leq k_{1}<k_{2}<\cdots<k_{2 m-1}<2 N$ satisfying (2.20) and (2.21), or equivalently, to calculate (2.8) for such $\varepsilon \in$ $\{0,1\}^{2 N}$ that

$$
|\{r \geq h: \varepsilon(r)=0\}| \leq|\{r \geq h: \varepsilon(r)=1\}|, \quad \forall h=1,2, \ldots, 2 N,
$$

and

$$
|\{r: \varepsilon(r)=0\}|=|\{r: \varepsilon(r)=1\}| .
$$

We shall denote, by $\{0,1\}_{+}^{2 N}$, the totality of such $\varepsilon \in\{0,1\}^{2 N}$ that satisfy $\left(2.23\right.$ a,b) and also by $\{0,1\}_{-}^{2 N}$ the subset $\{0,1\}^{2 N} \backslash\{0,1\}_{+}^{2 N}$.

By the definition of the creation operator, the vector (2.22) is equal to

$$
\prod_{h=k_{0}+1}^{k_{1}} A\left(g_{h}\right) \cdot \prod_{h=k_{1}+1}^{k_{2}} A^{+}\left(g_{h}\right) \cdots \prod_{h=k_{2 m-2}+1}^{k_{2 m-1}} A\left(g_{h}\right)\left[g_{k_{2 m-1}+1} \odot \cdots \odot g_{k_{2 m}}\right]
$$


This is, by Lemma (2.8), equal to

$$
\begin{gathered}
\prod_{h=k_{0}+1}^{k_{1}} A\left(g_{h}\right) \cdot \prod_{h=k_{1}+1}^{k_{2}} A^{+}\left(g_{h}\right) \ldots \prod_{h=k_{2 m-3}+1}^{k_{2 m-2}} A^{+}\left(g_{h}\right) \\
V_{k_{2 m}-2 k_{2 m-1}+k_{2 m-2}}\left(g_{k_{2 m-2}+1}, \ldots, g_{k_{2 m-1}} ; g_{k_{2 m-1}+1} \odot \cdots \odot g_{k_{2 m}}\right)
\end{gathered}
$$

By repeating the above procedure, one is able to rewrite the expression (2.11) to

$$
\begin{gathered}
V_{k_{2 m}-2 \sum_{j=0}^{m-1} k_{2 j+1}+\sum_{j=1}^{m-1} k_{2 j}}\left(g_{1}, \ldots, g_{k_{1}} ; g_{k_{1}+1} \odot \cdots \odot g_{k_{2}}\right. \\
\odot V_{k_{2 m}-2 \sum_{j=1}^{m-1} k_{2 j+1}+\sum_{j=2}^{m-1} k_{2 j}}\left(g_{k_{2}+1}, \ldots, g_{k_{3}} ; g_{k_{3}+1} \odot \cdots \odot g_{k_{4}}\right. \\
\left.\left.\odot \cdots \odot \cdots \odot V_{k_{2 m}-2 k_{2 m-1}+k_{2 m-2}}\left(g_{k_{2 m-2}+1}, \ldots, g_{k_{2 m-1}} ; g_{k_{2 m-1}+1} \odot \cdots \odot g_{k_{2 m}}\right) \cdots\right)\right)
\end{gathered}
$$

The expression (2.26) has a quite complicate form. We shall try to give another formula to express the quantity (2.11). In order to do this, we should first recall some basic facts about pair partitions on $2 \mathrm{~N}$ points.

For each $\varepsilon \in\{0,1\}^{2 N}$, there are an $m \leq N$ and a set $1 \leq l_{1}<l_{2}<\cdots<l_{m}$ $\leq 2 N$, such that

$$
\left\{l_{h}\right\}_{h=1}^{m}=\{k: \varepsilon(k)=0\} .
$$

It is clear that the $m \in \mathbf{N}$ and the ordered set $\left\{l_{h}\right\}_{h=1}^{m}$ are determined uniquely by the $\varepsilon$. In the following, the set $\left\{l_{h}\right\}_{h=1}^{m}$ will be called the left-index set of the $\varepsilon$. We have an equivalent statement of Lemma (2.9):

Lemma (2.10). $\varepsilon \in\{0,1\}_{+}^{2 N}$ if and only if its left-index set $\left\{l_{h}\right\}_{h=1}^{m}$ satisfies that:

1) $m=N, l_{1}=1, l_{N}<2 N$

2) for any $k=1,2, \ldots, 2 N$

$$
\begin{gathered}
\left.\mid\{k, k+1, \cdots, 2 N\} \cap\left\{l_{h}\right\}_{h=1}^{N}\right\} \mid \\
\left.\leq \mid\{k, k+1, \cdots, 2 N) \cap\left(\{1,2, \cdots, 2 N\} \backslash\left\{l_{h}\right\}_{h=1}^{N}\right)\right\} \mid
\end{gathered}
$$

Proof. The proof follows immediately from the equivalence between $\varepsilon \in$ $\{0,1\}_{+}^{2 N}$ and its left-index set.

For each $\varepsilon \in\{0,1\}_{+}^{2 N}$, with respect to its left-index set $\left\{l_{h}\right\}_{h=1}^{N}$, we can consider all pair partitions $\left\{\left(l_{h}, l_{h}^{\prime}\right)\right\}_{h=1}^{N}$. Where, $\left\{l_{h}^{\prime}\right\}_{h=1}^{N}:=\{1,2, \cdots, 2 N\} \backslash\left\{l_{h}\right\}_{h=1}^{N}$ must satisfy the following:

i) for any $h=1,2, \cdots, N, l_{h}^{\prime}>l_{h}$;

ii) for any $h, k=1,2, \cdots, N$ different, $l_{h}^{\prime}$ differs from $l_{k}^{\prime}$.

Of course, for each fixed left-index set $\left\{l_{h}\right\}_{h=1}^{N}$ (or equivalently, for each fixed 
$\varepsilon \in\{0,1\}_{+}^{2 N}$ ), there is at least one (possibly many) pair partition and the number of possible pair partitions depends on the left-index set. But we have:

Lemma (2.11). For each fixed $\varepsilon \in\{0,1\}_{+}^{2 N}$ (or equivalently, for each fixed leftindex set $\left.\left\{l_{h}\right\}_{h=1}^{N}\right)$, there is exactly one non-crossing pair partition $\left\{\left(l_{h}, l_{h}^{\prime}\right)\right\}_{h=1}^{N}$ which is determined by the usual non-crossing principle: for each $k<h$, if $l_{h}<l_{k}^{\prime}$, then $l_{h}^{\prime}$ $<l_{k}^{\prime}$.

Proof. The result is a consequence of the following two observations:

i) $\left\{\left(l_{h}, l_{h}^{\prime}\right)\right\}_{h=1}^{N}$ forms a non-crossing pair partition, only if $l_{N}^{\prime}=l_{N}+1$.

ii) $\left\{\left(l_{h}, l_{h}^{\prime}\right)\right\}_{h=1}^{N-1}$ forms a non-crossing pair partition of $\{1,2, \cdots, 2 N\} \backslash\left\{l_{N}, l_{N}^{\prime}\right\}$ if and only if $\left\{\left(l_{h}, l_{h}^{\prime}\right)\right\}_{h=1}^{N}$ forms a non-crossing pair partition of $\{1,2, \cdots, 2 N\}$.

In the following, the unique non-crossing pair partition determined by a given left-index set $\left\{l_{n}\right\}_{h=1}^{N}$ will be denoted by $\left\{\left(l_{h}, r_{h}\right)\right\}_{h=1}^{N}$ and the set $\left\{r_{h}\right\}_{h=1}^{N}$ will be called the right-index set.

For each given left-index set $\left\{l_{h}\right\}_{h=1}^{N}$, its right-index set $\left\{r_{h}\right\}_{h=1}^{N}$ is, in general, unordered and determined in such a way that: for any $p=0,1, \cdots, N-1$,

$$
r_{N-p}=\min \left\{k:\left(\{k, k+1, \cdots, 2 N\} \backslash\left\{l_{N-p+1}, \cdots, l_{N}\right\}\right) \subset\left\{r_{h}\right\}_{h=1}^{N}\right\}
$$

With the help of the results and terminologies given before, our main result in this section can be stated as:

ThEorem (2.12). The quantity (2.8) is equal to a possibly non zero value only if $n=2 N$ and $\varepsilon \in\{0,1\}_{+}^{2 N}$. In this case, there is a function of $N$-variables

$$
K\left(\left\{l_{h}, r_{h}\right\}_{h=1}^{N} ; y_{1}, \ldots, y_{N}\right)
$$

(where, $\left\{l_{h}, r_{h}\right\}_{h=1}^{N}$ is the left-right-index set of the $\varepsilon \in\{0,1\}_{+}^{2 N}$ ) such that the quantity (2.8) is equal to

$$
\int \mu\left(d y_{1}\right) \cdots \mu\left(d y_{N}\right) \prod_{h=1}^{N}\left(\bar{g}_{l_{h}}\left(y_{h}\right) g_{r_{h}}\left(y_{h}\right)\right) \cdot K\left(\left\{l_{h}, r_{h}\right\}_{h=1}^{N} ; y_{1}, \ldots, y_{N}\right)
$$

where, the function $K$ is determined uniquely by the $\varepsilon \in\{0,1\}_{+}^{2 N}$ and the interacting functions $\lambda_{1}, \lambda_{2}, \ldots, \lambda_{N}$. Moreover, if we define, on the space $M^{2}=M \times M$, the $\sigma$ - finite measure $\mu_{2}(d x, d y):=\mu(d x) \mu(d y) \delta(x-y)$. Then, the expression (2.28) can be represented as 


$$
\begin{gathered}
\int_{M^{2}} \mu_{2}\left(d x_{l_{1}}, d x_{r_{1}}\right) \cdots \int_{M^{2}} \mu_{2}\left(d x_{l_{N}}, d x_{r_{N}}\right) \prod_{h=1}^{N}\left(\bar{g}_{l_{h}}\left(x_{l_{h}}\right) g_{r_{h}}\left(x_{r_{h}}\right)\right) \\
\prod_{h=0}^{N-1} \frac{\lambda_{2 N-l_{N-h}-2 h}\left(\left\{x_{l_{N-h}+1}, \ldots, x_{2 N}\right\} \backslash\left\{x_{l_{p}}, x_{r_{p}}\right\}_{p=N-h+1}^{N}\right)}{\left.\lambda_{2 N-l_{N-h}-2 h-1}\left(\left\{x_{l_{N-h}+1}, \ldots, x_{2 N}\right\} \backslash\left\{x_{l_{p}}, x_{r_{p}}\right\}_{p=N-h+1}^{N} \cup\left\{x_{r_{N}-h}\right\}\right)\right)}
\end{gathered}
$$

where and hereinafter, for any $n \in \mathbf{N}, m \leq n$, any set $\left\{p_{h}\right\}_{h=1}^{m} \subset\{1,2, \cdots, n\}$ with cardinality $m$ and any function $F$ of $n-m$ vatiables, we have adopted the notation:

$$
F\left(\left\{x_{1}, x_{2}, \ldots, x_{n}\right\} \backslash\left\{x_{p_{h}}\right\}_{h=1}^{m}\right):=F\left(x_{1}, \ldots, \widehat{x_{p_{1}}}, \ldots, \widehat{x_{p_{m}}}, \ldots, x_{n}\right) .
$$

Proof. It is clear that we have only to prove the last conclusion.

For each given $\varepsilon \in\{0,1\}_{+}^{2 N}$, i.e. each given left-index set $\left\{l_{h}\right\}_{h=1}^{N}$, by Lemma (2.7), one knows that the vector

$$
A\left(g_{l_{N}}\right) A^{+}\left(g_{l_{N}+1}\right) \cdots A^{+}\left(g_{2 N}\right) \Phi=A\left(g_{l_{N}}\right)\left[g_{l_{N}+1} \odot \cdots \odot g_{2 N}\right]
$$

belongs to in $\mathscr{H}_{2 N-l_{N}-1}$ and

$$
\begin{gathered}
{\left[A\left(g_{l_{N}}\right)\left[g_{l_{N}+1} \odot \cdots \odot g_{2 N}\right]\right]\left(x_{l_{N}+2}, \ldots, x_{2 N}\right)} \\
=\left[g_{l_{N}+2} \odot \cdots \odot g_{2 N}\right]\left(x_{l_{N}+2}, \ldots, x_{2 N}\right) . \\
\int \mu\left(d x_{l_{N}}\right) \frac{\lambda_{2 N-l_{N}}\left(x_{l_{N}}, x_{l_{N}+2}, \ldots, x_{2 N}\right)}{\lambda_{2 N-l_{N}-1}\left(x_{l_{N}+2}, \ldots, x_{2 N}\right)}\left(\bar{g}_{l_{N}} g_{r_{N}}\right)\left(x_{l_{N}}\right) \\
=\left[g_{l_{N}+2} \odot \cdots \odot g_{2 N}\right]\left(x_{l_{N}+2}, \ldots, x_{2 N}\right) \cdot \\
\int_{M^{2}} \mu_{2}\left(d x_{l_{N}}, d x_{r_{N}}\right) \frac{\lambda_{2 N-l_{N}}\left(x_{l_{N}}, x_{l_{N}+2}, \ldots, x_{2 N}\right)}{\lambda_{2 N-l_{N}-1}\left(x_{l_{N}+2}, \ldots, x_{2 N}\right)} \bar{g}_{l_{N}}\left(x_{l_{N}}\right) g_{r_{N}}\left(x_{r_{N}}\right) .
\end{gathered}
$$

Since $r_{N}=l_{N}+1$, we are able to rewrite the right hand side of $(2.30)$ as

$$
\begin{gathered}
\bigodot_{\substack{l_{N}+1 \leq \alpha \leq 2 N \\
\alpha \notin\left\{r_{N}\right\}}} g_{\alpha} \int_{M^{2}} \mu_{2}\left(d x_{l_{N}}, d x_{r_{N}}\right) \cdot \\
\frac{\lambda_{2 N-l_{N}}\left(x_{l_{N}+1}, x_{l_{N}+2}, \ldots, x_{2 N}\right)}{\lambda_{2 N-l_{N}-1}\left(\left\{x_{l_{N}+1}, \ldots, x_{2 N}\right\} \backslash\left\{x_{r_{N}}\right\}\right)} \bar{g}_{l_{N}}\left(x_{l_{N}}\right) g_{r_{N}}\left(x_{r_{N}}\right) .
\end{gathered}
$$

It follows from the same arguments that

$$
A\left(g_{l_{N-1}}\right) A^{+}\left(g_{l_{N-1}+1}\right) \cdots A^{+}\left(g_{l_{N}-1}\right) A\left(g_{l_{N}}\right) A^{+}\left(g_{l_{N}+1}\right) \cdots A^{+}\left(g_{2 N}\right) \Phi
$$

$$
=A\left(g_{l_{N-1}}\right)\left[\bigodot_{\substack{l_{N-1}+1 \leq \alpha \leq 2 N \\ \alpha \notin\left\{l_{N}, r_{N}\right\}}} g_{\alpha}\right.
$$

$$
\left.\int_{M^{2}} \mu_{2}\left(d x_{l_{N}}, d x_{r_{N}}\right) \frac{\lambda_{2 N-l_{N}}\left(x_{l_{N}+1}, x_{l_{N}+2}, \ldots, x_{2 N}\right)}{\lambda_{2 N-l_{N}-1}\left(\left\{x_{l_{N}+1}, \ldots, x_{2 N}\right\} \backslash\left\{x_{r_{N}}\right\}\right)} \bar{g}_{l_{N}}\left(x_{l_{N}}\right) g_{r_{N}}\left(x_{r_{N}}\right)\right]
$$




$$
\begin{gathered}
=\bigodot_{\substack{l_{N-1}+1 \leq \alpha \leq 2 N \\
\alpha \notin\left\{r_{N-1}, l_{N}, r_{N}\right\}}} g_{\alpha} \int_{M^{2}} \mu_{2}\left(d x_{l_{N}-1}, d x_{r_{N-1}}\right) \bar{g}_{l_{N-1}}\left(x_{l_{N-1}}\right) g_{r_{N-1}}\left(x_{r_{N-1}}\right) \\
\frac{\lambda_{2 N-l_{N-1}-2}\left(\left\{x_{l_{N-1}+1}, \ldots, x_{2 N}\right\} \backslash\left\{x_{l_{N}}, x_{r_{N}}\right\}\right)}{\lambda_{2 N-l_{N-1}-3}\left(\left\{x_{l_{N-1}+1}, \ldots, x_{2 N}\right\} \backslash\left\{x_{r_{N-1}}, x_{l_{N}}, x_{r_{N}}\right\}\right)} \\
\quad \int_{M^{2}} \mu_{2}\left(d x_{l_{N}}, d x_{r_{N}}\right) \bar{g}_{l_{N}}\left(x_{l_{N}}\right) g_{r_{N}}\left(x_{r_{N}}\right) \frac{\lambda_{2 N-l_{N}}\left(x_{l_{N}+1}, x_{l_{N}+2}, \ldots, x_{2 N}\right)}{\lambda_{2 N-l_{N}-1}\left(\left\{x_{l_{N}+1}, \ldots, x_{2 N}\right\} \backslash\left\{x_{r_{N}}\right\}\right)} .
\end{gathered}
$$

By repeating the above arguments, the proof is completed.

\section{Remark.}

i) From the above Theorem, we know that in order to calculate the quantity (2.8), one must only consider non-crossing pair partitions. In other words, the creation and annihilation operators on the interacting Free Fock space still satisfy the non-crossing principle.

ii) The function $K$ is, in general, not a constant.

\section{§3. The deformed Gaussianity and the vacuum distribution of field operator}

In the present section we discuss some more properties of the creation and annihilation operators.

Creation and annihilation operators on the usual (uninteracting) Boson, Fermion and Free Fock spaces (over a certain Hilbert space $\mathscr{H}$ ) possess an important property: for any $n \in \mathbf{N}, g_{1}, \ldots, g_{n} \in \mathscr{H}$ and $\varepsilon \in\{0,1\}^{n}$, the vacuum expectation of the product

$$
A^{\varepsilon(1)}\left(g_{1}\right) \cdots A^{\varepsilon(n)}\left(g_{n}\right)
$$

vanishes certainly in the following two cases

i) $n$ is odd;

ii) $n=2 N$ for some $N \in \mathbf{N}$ but $\varepsilon \in\{0,1\}_{-}^{2 N}$.

In the case of $\varepsilon \in\{0,1\}_{+}^{2 N}$ with the left-index set $\left\{l_{h}\right\}_{h=1}^{N}$, the vacuum expectation of (3.1) has of form

$$
\sum_{\left\{l_{h}, l_{h}^{\prime}\right\}_{h=1}^{N} \in\{p . p .2 N\}} f\left(\left\{l_{h}, l_{h}^{\prime}\right\}_{h=1}^{N}\right) \prod_{h=1}\left\langle\Phi, A\left(g_{l_{h}}\right) A^{+}\left(g_{l_{h}^{\prime}}\right) \Phi\right\rangle
$$

Where and hereinafter, by $\{$ p.p. $2 N\}$, we denote the totality of all pair partitions of $\{1,2, \ldots, 2 N\}$. The $f\left(\left\{l_{h}, l_{h}^{\prime}\right\}_{h=1}^{N}\right)$ factor depends on the tensor structure:

i) $f\left(\left\{l_{h}, l_{h}^{\prime}\right\}_{h=1}^{N}\right)=1$, if we are considering the Boson case;

ii) $f\left(\left\{l_{h}, l_{h}^{\prime}\right\}_{h=1}^{N}\right)=(-1)^{\left\|\left\{l_{h}, l_{h}^{\prime}\right\}_{h=1}^{N}\right\|}$, if we are considering the Fermion case, 
where $\left\|\left\{l_{h}, l_{h}^{\prime}\right\}_{h=1}^{N}\right\|$ is the index of the permutation

$$
\{1,2,3,4, \ldots, 2 N-2,2 N\} \rightarrow\left\{l_{1}, l_{1}^{\prime}, l_{2}, l_{2}^{\prime}, \ldots, l_{N}, l_{N}^{\prime}\right\}
$$

iii) in the Free Fock case,

$$
f\left(\left\{l_{h}, l_{h}^{\prime}\right\}_{h=1}^{N}\right)= \begin{cases}1, & \text { if }\left\{l_{h}, l_{h}^{\prime}\right\}_{h=1}^{N} \in\{n \text {-c.p.p. } 2 N\} \\ 0, & \text { otherwise }\end{cases}
$$

where, here and in the following, by $\{n$-c.p.p. $2 N\}$ we denote the totality of all non-crossing pair partitions of $\{1,2, \ldots, 2 N\}$.

Roughly speaking, in all the above three cases, any $2 N-1$ points function is equal to zero and any $2 N$ points function equal a sum of products of two points functions. In other word, under the vacuum state, the creation and annihilation operators possess the Gaussianity.

Now let us see the interacting Free Fock space. It is clear that under the vacuum state, creation and annihilation operators do not, in general, possess the above property. For example, in the case of $N=2$, according to the results in the section 2 , the expression

$$
\left\langle\Phi, A\left(g_{1}\right) A\left(g_{2}\right) A^{+}\left(g_{3}\right) A^{+}\left(g_{4}\right) \Phi\right\rangle
$$

is equal to

$$
\int \mu(d x) \mu(d y)\left(\bar{g}_{2} \cdot g_{3}\right)(x)\left(\bar{g}_{1} \cdot g_{4}\right)(y) \lambda_{2}(x, y)
$$

and which is in general impossible to be represented to a form like

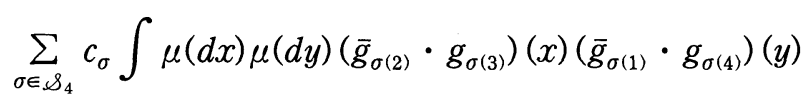

where, by $\mathscr{S}_{n}$, we denote the $n$-permutation group. Therefore, under the vacuum state, the creation and annihilation operators do not, in general, possess the Gaus sianity.

But, on the interacting Free Fock space, under the vacuum state, the creation and annihilation operators possess some properties similar to the Gaussianity. In order to look it, let us introduce some notations.

Definition (3.1) For each $n, m \in \mathbf{Z}, k \in \mathbf{N}$ and a function $f$ defined on the set $\{n+1, n+2, \ldots, n+m\}$, the $\pm k$-shift of the function $f$ is a function defined on the set $\{n+1 \pm k, n+2 \pm k, \ldots, n+m \pm k\}$ : 


$$
\left(u_{ \pm}^{k} f\right)(h \pm k):=f(h), \quad \forall h \in\{n+1, n+2, \ldots, n+m\}
$$

With this terminology, we can state a simple result:

Lemma (3.2). For each $N \in \mathbf{N}, p<N$ and $\varepsilon \in\{0,1\}_{+}^{2 N}$ (with the left-rightindex set $\left.\left\{l_{h}, r_{h}\right\}_{h=1}^{N}\right)$, if we define a map $\epsilon$, which takes value in $\{0,1\}$, by

$$
\left.\epsilon\right|_{\left\{1,2, \cdots, l_{p}-1\right\}}:=\left.\varepsilon\right|_{\left\{1,2, \cdots, l_{p}-1\right\}}
$$

$\epsilon\left(l_{p}\right):=\varepsilon\left(r_{p}+1\right), \quad \epsilon\left(l_{p}+1\right):=\varepsilon\left(r_{p}+2\right), \ldots, \epsilon\left(2 N+l_{p}-r_{p}-1\right):=\varepsilon(2 N)$

then, $\epsilon \in\{0,1\}_{+}^{2 \frac{2 N-l_{p}-r_{p}-1}{2}}$

Proof. First of all, one has to show that $2 N-l_{p}-r_{p}-1$ is even. In fact, by the non-crossing principle, if $l_{d} \in\left(l_{p}, r_{p}\right)$, then it is certainly true that $r_{d} \in$ $\left(l_{p}, r_{p}\right)$. Therefore, $r_{p}-l_{p}-1(\leq 2 N)$ is always even, and $2 N-l_{p}-r_{p}-1$ so is.

Again by the non-crossing principle, the restriction of the given $\varepsilon \in$ $\{0,1\}_{+}^{2 N}$ on the set $\{1,2, \ldots, 2 N) \backslash\left\{l_{p}, l_{p}+1, \cdots, r_{p}\right\}$ permits a unique non-crossing pair partition $\left\{\left(l_{h}, r_{k}\right)\right\} h \in\{1,2, \ldots, N\} \backslash\left\{d: l_{p} \leq l_{d} \leq r_{p}\right\}$. So the thesis is obtained.

Let us now examine the quantity (2.8) for $n=2 N$ and $\varepsilon \in\{0,1\}_{+}^{2 N}$. It is obvious that $l_{1}=1, r_{1}$ is even and

$$
\left.\varepsilon\right|_{\left\{1,2, \cdots, r_{1}\right\}} \in\{0,1\}_{+}^{2 \frac{r_{1}}{2}} .
$$

By the non-crossing principle, $r_{1}+1$ must be a left-index, say $l_{d_{1}}$. Moreover, we know that $r_{d_{1}}-l_{d_{1}}-r_{1}+1$ is even, $r_{d_{1}}+1$ is a left index and

$$
\left.\left(u_{-}^{r_{1}} \varepsilon\right)\right|_{\left\{1,2, \cdots, r_{d_{1}}-l_{d_{1}}+1-r_{1}\right\}} \in\{0,1\}_{+}^{2 \frac{r_{d_{1}}-l_{d_{1}}-r_{1}+1}{2}} .
$$

By repeating the argument, having obtained the pices

$$
1, \ldots, r_{1},\left(r_{1}+1=\right) l_{d_{1}}, \ldots, r_{d_{1}},\left(r_{d_{1}}+1=\right) l_{d_{2}}, \ldots, r_{d_{2}}, \ldots\left(r_{d_{i-1}}+1=\right) l_{d_{i}}, \ldots, r_{d_{i}}
$$

since the $r_{d_{i}}+1$ must be a left-index, say $l_{d_{i+1}}$, we have the next pice

$$
l_{d_{i+1}}, l_{d_{i+1}}+1, \ldots, r_{d_{i+1}}+1
$$

Thus, in fact we have proved that

Lemma (3.3). Each $\varepsilon \in\{0,1\}_{+}^{2 N}$ determines uniquely an $m \leq N, 1 \leq N_{1}, \ldots$, $N_{m} \leq N$ and $\varepsilon_{i} \in\{0,1\}_{+}^{2 N_{i}}(i=1,2, \ldots, m)$ such that $\sum_{i=1} N_{i}=N$ and 


$$
\left.\left(u_{-}^{2 N_{j}} \varepsilon\right)\right|_{\left\{2 N_{j}+1,2 N_{j}+2, \cdots, 2 N_{j+1}\right\}}=\varepsilon_{\imath} .
$$

Thank to this result, we find immediately that

Lemma (3.4). The quantity (2.8) for $n=2 N$ and $\varepsilon \in\{0,1\}_{+}^{2 N}$ is equal to

$$
\left\langle\Phi, \prod_{h=1}^{r_{1}} A^{\varepsilon(h)}\left(g_{h}\right) \prod_{h=l_{d_{1}}}^{r_{d_{1}}} A^{\varepsilon(h)}\left(g_{h}\right) \cdots \prod_{h=l_{d_{m}}}^{r_{d m}} A^{\varepsilon(h)}\left(g_{h}\right) \Phi\right\rangle
$$

where,

i) $1 \leq m \leq N, d_{,} \leq N,(j=1,2, \ldots, m), d_{m}=N$ and they are determined uniquely by the given $\varepsilon$;

ii) $l_{d_{1}}=r_{1}+1, l_{d_{2}}=r_{d_{1}}+1, \ldots, l_{d_{m}}=r_{d_{m-1}}+1$.

Remark. In the language of diagram, each $\varepsilon \in\{0,1\}_{+}^{2 N}$ permits a unique non-crossing diagram with the vertices $\{1,2, \ldots, 2 N\}$. Any leg in the diagram connects two vertices $l_{h}, r_{h}(h=1, \ldots, N)$. The decomposition stated in Lemma (3.3) means that the diagram determined by $\varepsilon \in\{0,1\}_{+}^{2 N}$ can be decomposited into $m$ pices with the following properties:

i) there is not any leg connecting two vertices from two different pices. In other word, for any $l_{h}, l_{k}$ in two different pices, if $l_{h}<l_{k}$, then $r_{h}<l_{h}$;

ii) in any pice, the first vertex is paired to the last vertex;

iii) the restriction of the full diagram (determined by the given $\varepsilon \in\{0,1\}_{+}^{2 N}$ ) on each pice gives a non-crossing diagram on the set of the corresponding vertices.

In the following. we shall call an $\varepsilon \in\{0,1\}_{+}^{2 N}$ total-connected if $r_{1}=2 N$ and call the decomposition (stated in the above) the total-connected decomposition of the given $\varepsilon \in\{0,1\}_{+}^{2 N}$.

Now we are ready to state what we call the deformed Gaussianity.

Lemma (3.5). For each $\varepsilon \in\{0,1\}_{+}^{2 N}$ with the total-connected composition given in Lemma (3.4), the expression (3.5) is equal to

$$
\left\langle\Phi, \prod_{h=1}^{r_{1}} A^{\varepsilon(h)}\left(g_{h}\right) \Phi\right\rangle \cdot\left\langle\Phi, \prod_{h=l_{d_{1}}}^{r_{d_{1}}} A^{\varepsilon(h)}\left(g_{h}\right) \Phi\right\rangle \cdots\left\langle\Phi, \prod_{h=l_{d_{m}}}^{r_{d_{m}}} A^{\varepsilon(h)}\left(g_{h}\right) \Phi\right\rangle .
$$

Proof. By the definition of the creation and annihilation operators, for any vector $G$ in a certain $\mathscr{H}_{n}$, any $f, f_{1}, \ldots, f_{n} \in \mathscr{H}$, and any $\varepsilon \in\{0,1\}^{n}$, the action of operator $\Pi_{h=1}^{n} A^{\varepsilon(h)}\left(f_{h}\right) A(f)$ on the vector $G$ is equal to that of the operator 
$\Pi_{h=1}^{n} A^{\varepsilon(h)}\left(f_{h}\right)$ on the vector $A(f) G$, i.e.

$$
\left[\prod_{h=1}^{n} A^{\varepsilon(h)}\left(f_{h}\right) A(f)\right] G=\prod_{h=1}^{n} A^{\varepsilon(h)}\left(f_{h}\right)[A(f) G] .
$$

Hence, the quantity (3.5) is equal to

$$
\left\langle\Phi, \prod_{h=1}^{r_{1}} A^{\varepsilon(h)}\left(g_{h}\right) \cdot \prod_{h=l_{d_{1}}}^{r_{d_{1}}} A^{\varepsilon(h)}\left(g_{h}\right) \cdots \prod_{h=l_{d_{m-1}}}^{r_{d_{m p-1}}} A^{\varepsilon(h)}\left(g_{h}\right)\left[\prod_{h=l_{d_{m}}}^{r_{d m}} A^{\varepsilon(h)}\left(g_{h}\right) \Phi\right]\right\rangle .
$$

In (3.7), since $\left\{l_{d_{m}}, l_{d_{m}}+1, \ldots, r_{d_{m}}\right\}$ is a total-connected pice determined by the $\varepsilon$, the vector $\Pi_{h=l_{d_{m}}}^{r_{d_{m}}} A^{\varepsilon(h)}\left(g_{h}\right) \Phi$ must be a number, i.e. it belongs to $\mathscr{H}_{0}=\mathbf{C}$. This argument guarantees that (3.5) is equal to

$$
\left\langle\Phi, \prod_{h=1}^{r_{1}} A^{\varepsilon(h)}\left(g_{h}\right) \cdot \prod_{h=l_{d_{1}}}^{r_{d_{d}}} A^{\varepsilon(h)}\left(g_{h}\right) \cdots \prod_{h=l_{d_{m-1}}}^{r_{d}-1} A^{\varepsilon(h)}\left(g_{h}\right) \Phi\right\rangle \cdot\left\langle\Phi, \prod_{h=l_{d_{m}}}^{r_{d}} A^{\varepsilon(h)}\left(g_{h}\right) \Phi\right\rangle .
$$

By repeating the above argument to the first scalar product in (3.8) and applying the induction, we finish the demonstration.

The result of Lemma (3.5) could be stated in a simple way: for any $\varepsilon \in$ $\{0,1\}_{+}^{2 N}$, the vacuum expectation of the operator

$$
\prod_{h=1}^{2 N} A^{\varepsilon(h)}\left(g_{h}\right)
$$

is equal to a product of some scalars. Moreover, in the product, each factor is equal to the vacuum expectation of a sub-product of (3.9) and the indices of the operators in a sub-product belong to a certain total-connected pices of the given $\varepsilon$.

By this result, in order to calculate a quantity like (3.5), one has only to do the calculation for $\varepsilon \in\{0,1\}_{+}^{2 N}$ being total-connected, i.e. $r_{1}=2 N$. But unfortunately, on such calculation, we have few thing to say more than the results obtained in the section 2. Of course, in some special cases, one can get very clear expression of the quantity like (3.5) even for $\varepsilon \in\{0,1\}_{+}^{2 N}$ being total-connected and such results will be presented in the next section.

Now, we are going to compute the vacuum moments of an operator with the form $A(g)+A^{+}(g)$. Obviously, this is equivalent to calculate the vacuum moments of the field operator $\frac{1}{2}\left(A(g)+A^{+}(g)\right)$.

It is easy to see that for any $n$ odd, the $n$-th vacuum moment of the operator $A(g)+A^{+}(g)$ is equal to zero. So, we shall assume that $n=2 N$ for some $N \in \mathbf{N}$. By expanding the power $\left(A(g)+A^{+}(g)\right)^{2 N}$, one has that 


$$
\left\langle\Phi,\left(A(g)+A^{+}(g)\right)^{2 N} \Phi\right\rangle=\sum_{\varepsilon \in\{0,1\}^{2 N}}\left\langle\Phi, \prod_{h=1}^{2 N} A^{\varepsilon(h)}(g) \Phi\right\rangle .
$$

Thank to the results obtained in the section 2 , the scalar product in the right hand side of (3.10) is possibly not equal to zero only if $\varepsilon \in\{0,1\}_{+}^{2 N}$. Therefore, the $2 N$-th vacuum moment is equal to

$$
u_{N}:=\sum_{\varepsilon \in\{0,1\}_{+}^{2 N}}\left\langle\Phi, \prod_{h=1}^{2 N} A^{\varepsilon(h)}(g) \Phi\right\rangle .
$$

By Lemma (3.5), the scalar product in (3.11) is equal to a product of some numbers. In the product, each number is nothing else but the vacuum expectation of a sub-product of (3.9) and the indices of the operators in the sub-product belong to a certain total-connected pices of the given $\varepsilon$. Now, define

$$
v_{m}:=\sum_{\substack{\varepsilon \in\{0,1\}^{2 m} \\ \varepsilon \text { is total-connected }}}\left\langle\Phi, \prod_{h=1}^{2 m} A^{\varepsilon(h)}(g) \Phi\right\rangle, \quad m=0,1, \ldots
$$

One gets easily that

$$
u_{0}=v_{0}=u_{1}=v_{1}=1
$$

and moreover,

Lemma (3.6). With the above notations, for any $n=2,3, \ldots$,

$$
u_{n}=\sum_{k=1}^{n} v_{k} u_{n-k}
$$

Proof. In order to prove (3.14), we reconsider the sum in the (3.11) for all $\varepsilon \in\{0,1\}_{+}^{2 N}$ according to the possible length of the first total-connected pice, i.e. the possible value of $r_{1}$. It is clear that $r_{1}$ takes value in $\{2,4, \ldots, 2(N-1), 2 N\}$. Hence, $u_{n}$ is equal to

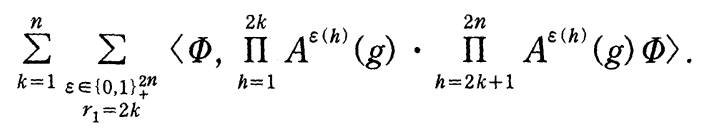

By Lemma (3.5), the scalar product in (3.15) is equal to

$$
\left\langle\Phi, \prod_{h=1}^{2 k} A^{\varepsilon(h)}(g) \Phi\right\rangle \cdot\left\langle\Phi, \prod_{h=2 k+1}^{2 n} A^{\varepsilon(h)}(g) \Phi\right\rangle
$$

For each fixed $k=1,2, \ldots, n$, since $r_{1}=2 k$, it is clear that if one defines 


$$
\begin{gathered}
\varepsilon_{1}:=\left.\varepsilon\right|_{\{1,2, \ldots, 2 k\}} \\
\varepsilon_{2}:=u_{-}^{2 k} \varepsilon
\end{gathered}
$$

$$
\text { Moreover, as } \varepsilon \text { running over the set }\{\varepsilon \in
$$
then, $\varepsilon_{1} \in\{0,1\}_{+}^{2 k}, \varepsilon_{2} \in\{0,1\}_{+}^{2(n-k)}$. Moreover, as $\varepsilon$ running over the set $\{\varepsilon \in$ $\left.\{0,1\}_{+}^{2 n}: r_{1}=2 k\right\}, \varepsilon_{1}$ runs over $\{0,1\}_{+}^{2 k}$ and $\varepsilon_{2}$ over $\{0,1\}_{+}^{2(n-k)}$. So, (3.15) is equal to

$$
\begin{gathered}
\sum_{k=1}^{n} \sum_{\substack{\varepsilon \in\{0,1\}^{2} \\
r_{1}=2 k}}\left\langle\Phi, \prod_{h=1}^{2 k} A^{\varepsilon(h)}(g) \cdot \prod_{h=2 k+1}^{2 n} A^{\varepsilon(h)}(g) \Phi\right\rangle \\
=\sum_{k=1}^{n} \sum_{\substack{\varepsilon \in\{0,1\}_{+}^{2 n} \\
r_{1}=2 k}}\left\langle\Phi, \prod_{h=1}^{2 k} A^{\varepsilon(h)}(g) \Phi\right\rangle \cdot\left\langle\Phi, \prod_{h=2 k+1}^{2 n} A^{\varepsilon(h)}(g) \Phi\right\rangle
\end{gathered}
$$

and which is equal to

$$
\sum_{k=1}^{n} \sum_{\varepsilon_{1} \in\{0,1\}_{+}^{2 k}}\left\langle\Phi, \prod_{h=1}^{2 k} A^{\varepsilon_{1}(h)}(g) \Phi\right\rangle \cdot \sum_{\varepsilon_{2} \in\{0,1)_{+}^{(n-k)}}\left\langle\Phi, \prod_{h=1}^{2(n-k)} A^{\varepsilon_{2}(h)}(g) \Phi\right\rangle .
$$

This is nothing else but the right hand side of (3.14).

Remark. A trivial conclusion of Lemma (3.6) is that on the usual Free Fock space, the formula (3.14) is certainly valid. Moreover, in that case, any totalconnected pice is controlable: In fact, $v_{k}=u_{k-1}$. Therefore, on the usual Free Fock spae, (3.14) takes the form

$$
u_{n}=\sum_{k=1}^{n} u_{k-1} u_{n-k}, \quad u_{0}=u_{1}=1
$$

As the end of the section, we give a result which can be easily proved by expanding the operator $\left(A(g)+A^{+}(g)\right)^{n}$ as a sum of products of the creation, annihilation operators and applying Theorem (2.12).

Theorem (3.7). For any $n \in \mathbf{N}, g \in \mathscr{H}$, the vacuum expectation of the operator $\left(A(g)+A^{+}(g)\right)^{n}$ is equal to zero if $n$ odd; if $n=2 N$, the vacuum expectation of $\left(A(g)+A^{+}(g)\right)^{n}$ is given by

$$
\begin{aligned}
& \sum_{\left\{\left(l_{h}, r_{h}\right)\right\}_{h=1}^{N} \in\{n-\text { c.p. . } 2 N\}} \int_{M^{2}} \mu_{2}\left(d x_{l_{1}}, d x_{r_{1}}\right) \cdots \int_{M^{2}} \mu_{2}\left(d x_{l_{N}}, d x_{r_{N}}\right) \prod_{h=1}^{N}\left(\bar{g}_{l_{h}}\left(x_{l_{h}}\right) g_{r_{h}}\left(x_{r_{h}}\right)\right) \\
& \quad \prod_{h=0}^{N-1} \frac{\lambda_{2 N-l_{N-h}-2 h}\left(\left\{x_{l_{N-h}+1}, \ldots, x_{2 N}\right\} \backslash\left\{x_{l_{p}}, x_{r_{p}}\right\}_{p=N-h+1}^{N}\right)}{\lambda_{2 N-l_{N-h}-2 h-1}\left(\left\{x_{l_{N-h}+1}, \ldots, x_{2 N}\right\} \backslash\left(\left\{x_{l_{p}}, x_{r_{p}}\right\}_{p=N-h+1}^{N} \cup\left\{x_{r_{N-h}}\right\}\right)\right)} .
\end{aligned}
$$




\section{§4. Examples and applications}

In this section, we present some examples of the interacting Free Fock space.

The first example is that the interacting functions $\lambda_{1}, \lambda_{2}, \ldots$ possess the following forms: there exist functions $\left\{\alpha_{n, k}\right\}_{1 \leq k \leq n<\infty}$, such that

$$
\lambda_{n}\left(x_{1}, \ldots, x_{n}\right)=\alpha_{n, 1}\left(x_{1}\right) \cdot \alpha_{n, 2}\left(x_{2}\right) \cdot \cdots \cdot \alpha_{n, n}\left(x_{n}\right) .
$$

A special interesting case is that

(4.1) $M=[a, b], \quad$ with $0 \leq a<b, \quad \alpha_{n, k}\left(x_{k}\right)=\frac{\left(x_{k}-a\right)^{(k-1) p}}{(b-a)^{(k-1) p}}, \quad$ with a $p \geq 0$.

That is

$$
\begin{gathered}
\lambda_{1}=1, \quad \lambda_{2}\left(x_{1}, x_{2}\right)=\frac{\left(x_{2}-a\right)^{p}}{(b-a)^{p}}, \ldots, \\
\lambda_{n}\left(x_{1}, x_{2}, \ldots, x_{n}\right)=\frac{\left(x_{2}-a\right)^{p}}{(b-a)^{p}} \frac{\left(x_{3}-a\right)^{2 p}}{(b-a)^{2 p}} \cdots \frac{\left(x_{n}-a\right)^{(n-1) p}}{(b-a)^{(n-1) p}} .
\end{gathered}
$$

Of course, with these interacting functions $\left\{\lambda_{n}\right\}_{n=1}^{\infty}$, we can construct an interacting Free Fock space as before, which is the usual Free Fock space if and only if $p=0$.

For such interacting functions $\left\{\lambda_{n}\right\}_{n=1}^{\infty}$, the vacuum expectation (3.5) can be handled even for $p>0$. The main idea to do this is to notice the following fact: If the interacting functions $\left\{\lambda_{n}\right\}_{n=1}^{\infty}$ are given as in (4.1), then for any $n \in \mathbf{N}, f, f_{1}$, $f_{2}, \ldots, f_{n} \in \mathscr{H}=L^{2}([a, b], d \mu)$, by Lemma $(2.7)$

$$
\begin{gathered}
{\left[A(f) A^{+}\left(f_{1}\right) \cdots A^{+}\left(f_{n}\right) \Phi\right]\left(x_{2}, \ldots, x_{n}\right)} \\
=\left(f_{2} \odot \cdots \odot f_{n}\right)\left(x_{2}, \ldots, x_{n}\right) \int_{a}^{b} \mu(d x)\left(\bar{f} \cdot f_{1}\right)(x) \frac{\lambda_{n}\left(x, x_{2}, \ldots, x_{n}\right)}{\lambda_{n-1}\left(x_{2}, \ldots, x_{n}\right)}
\end{gathered}
$$

$$
=\left(f_{2} \odot \cdots \odot f_{n}\right)\left(x_{2}, \ldots, x_{n}\right) \int_{a}^{b} \mu(d x)\left(\bar{f} \cdot f_{1}\right)(x) \frac{\frac{\left(x_{2}-a\right)^{p}}{(b-a)^{p}} \frac{\left(x_{3}-a\right)^{2 p}}{(b-a)^{2 p}} \cdots \frac{\left(x_{n}-a\right)^{(n-1) p}}{(b-a)^{(n-1) p}}}{\frac{\left(x_{3}-a\right)^{p}}{(b-a)^{p}} \frac{\left(x_{4}-a\right)^{2 p}}{(b-a)^{2 p}} \cdots \frac{\left(x_{n}-a\right)^{(n-2) p}}{(b-a)^{(n-2) p}}}
$$

$=\left(f_{2} \odot \cdots \odot f_{n}\right)\left(x_{2}, \ldots, x_{n}\right) \int_{a}^{b} \mu(d x)\left(\bar{f} \cdot f_{1}\right)(x) \cdot \frac{\left(x_{2}-a\right)^{p}}{(b-a)^{p}} \cdot \frac{\left(x_{3}-a\right)^{p}}{(b-a)^{p}} \cdots \frac{\left(x_{n}-a\right)^{p}}{(b-a)^{p}}$

$$
=\left(f_{2} \cdot I_{p}\right) \odot \cdots \odot\left(f_{n} \cdot I_{p}\right)\left(x_{2}, \ldots, x_{n}\right) \int_{a}^{b} \mu(d x)\left(\bar{f} \cdot f_{1}\right)(x)
$$


where, $I_{p}$ is the map on $[a, b]$ defined as: $I(x):=\frac{(x-a)^{p}}{(b-a)^{p}}$. Again by Lemma (2.7),

$$
\begin{gathered}
{\left[A(f) A^{+}\left(f_{1}\right) \cdots A^{+}\left(f_{n-1}\right) \Phi\right]\left(x_{2}, \ldots, x_{n-1}\right)} \\
=\left(f_{2} \odot \cdots \odot f_{n-1}\right)\left(x_{2}, \ldots, x_{n-1}\right) \int_{a}^{b} \mu(d x)\left(\bar{f} \cdot f_{1}\right)(x) \frac{\lambda_{n-1}\left(x, x_{2}, \ldots, x_{n-1}\right)}{\lambda_{n-2}\left(x_{2}, \ldots, x_{n-1}\right)}
\end{gathered}
$$

$$
\begin{aligned}
=\left(f_{2} \odot \cdots \odot f_{n-1}\right)\left(x_{2}, \ldots, x_{n-1}\right) \int_{a}^{b} \mu( & d x)\left(\bar{f} \cdot f_{1}\right)(x) \\
& \frac{\frac{\left(x_{2}-a\right)^{p}}{(b-a)^{p}} \frac{\left(x_{3}-a\right)^{2 p}}{(b-a)^{2 p}} \cdots \frac{\left(x_{n-1}-a\right)^{(n-2) p}}{(b-a)^{(n-2) p}}}{\frac{\left(x_{3}-a\right)^{p}}{(b-a)^{p}} \frac{\left(x_{4}-a\right)^{2 p}}{(b-a)^{2 p}} \cdots \frac{\left(x_{n-1}-a\right)^{(n-2) p}}{(b-a)^{(n-3) p}}}
\end{aligned}
$$

$$
=\left(f_{2} \odot \cdots \odot f_{n-1}\right)\left(x_{2}, \ldots, x_{n-1}\right) \int_{a}^{b} \mu(d x)\left(\bar{f} \cdot f_{1}\right)(x) \frac{\left(x_{2}-a\right)^{p}}{(b-a)^{p}} .
$$

$$
\begin{array}{r}
\frac{\left(x_{3}-a\right)^{p}}{(b-a)^{p}} \cdots \frac{\left(x_{n-1}-a\right)^{p}}{(b-a)^{p}} \\
=\left(f_{2} \cdot I_{p}\right) \odot \cdots \odot\left(f_{n-1} \cdot I_{p}\right)\left(x_{2}, \ldots, x_{n-1}\right) \int_{a}^{b} \mu(d x)\left(\bar{f} \cdot f_{1}\right)(x)
\end{array}
$$

This means that the influences to the part of $A^{+}\left(f_{1}\right), \ldots, A^{+}\left(f_{n-1}\right)$ in the following two actions:

$$
A(f) A^{+}\left(f_{1}\right) \cdots A^{+}\left(f_{n}\right) \Phi
$$

and

$$
A(f) A^{+}\left(f_{1}\right) \cdots A^{+}\left(f_{n-1}\right) \Phi
$$

are the same. And moreover, each procedure of pairing an annihilation operator to a creation operator (according to the non-crossing principle) contributes a map $I_{p}$ to the last creation operator. Thus, in fact we have proved the following result:

Lemma (4.1). For any $n \in \mathbf{N}, g_{1}, \ldots, g_{2 n} \in \mathscr{H}$ and $\varepsilon \in\{0,1\}_{+}^{2 n}$ totalconnected, the vacuum expectation

$$
\left\langle\Phi, \prod_{h=1}^{2 n} A^{\varepsilon(h)}\left(g_{h}\right) \Phi\right\rangle
$$

is equal to 


$$
\left\langle\Phi, \prod_{h=2}^{2(n-1)} A^{\varepsilon(h)}\left(g_{h}\right) \Phi\right\rangle \cdot\left\langle\Phi, A\left(g_{1}\right) A\left(g_{2 n} I_{p}^{n-1}\right) \Phi\right\rangle
$$

where, for any $k \in \mathbf{N}$.

Remark. It seems true that from the central limit of the quantum Bernoulli processes, one could find an interacting Free Fock space with the interacting functions given by (4.1).

It is interesting to calculate the above expectation for $g_{h}=1$. Moreover, up to a trivial linear transformation, we shall assume in the following that $a=0, b=$ 1. In this case, we also assume that $\mu$ is the Lebesgue measure.

Lemma (4.2). For any $n \in \mathbf{N}$ and $p \geq 0$, let us define

$$
U_{n, p}:=\left\langle\Phi,\left(A(1)+A^{+}(1)\right)^{2 n} \Phi\right\rangle
$$

Then,

$$
U_{0, p}=U_{1, p}=1, \quad U_{n, p}=\sum_{k=0}^{n-1} \frac{1}{1+p k} U_{k, p} U_{n-k-1, p}
$$

Proof. Thank to Lemma (4.1), Lemma (3.6) and the above discussions, in order to prove the present lemma, it is sufficient to show that

$$
\left\langle\Phi, A(1) A\left(I_{p}^{k}\right) \Phi\right\rangle=\frac{1}{1+p k} .
$$

But this requires only a trivial computation.

An important problem is to know the density function (if it exists) of the random variable corresponding to the operator $A(1)+A^{+}(1)$. The density function (if it exists) will depend on the parameter $p \geq 0$ and be equal to the density function of the Wigner distribution on $(-2,2)$. Just by this reason, we shall call the distribution of the random variable corresponding to the operator $A(1)+$ $A^{+}(1)$ as the deformed Wigner distribution.

In order to know more informations on the distribution, we do some analysis. First of all,

Lemma (4.3). For any $p \geq 0, n=0,1,2, \ldots$, 


$$
U_{n, p} \leq U_{n, 0}
$$

Proof. For $n=0,1$, the inequality (4.9) takes equality. If (4.9) is true for $n \leq m$,

then

$$
U_{m+1, p}=\sum_{k=0}^{m} \frac{1}{1+p k} U_{k, p} U_{m-k, p} \leq \sum_{k=0}^{m} \frac{1}{1+p k} U_{k, 0} U_{m-k, 0} \leq \sum_{k=0}^{m} U_{k, 0} U_{m-k, 0}=U_{m+1,0}
$$

The induction guarantees the thesis.

Lemma (4.4). For any $p \geq 0$, the generating function of $\left\{U_{n, p}\right\}_{n=0}^{\infty}$

$$
E_{p}(z):=\sum_{n=0}^{\infty} U_{n, p} z^{n}
$$

is well defined on a neighborhood of the origin. Moreover, for each $n \in \mathbf{N}$

$$
U_{n, p}=\frac{1}{n !} E_{p}^{(n)}(0)
$$

and $E_{p}(z)$ satisfies the following integral equation:

$$
E_{p}(z)=1+z \cdot E_{p}(z) \int_{0}^{1} E_{p}\left(y^{p} z\right) d y
$$

Proof. (4.11) is an easy consequence of (4.10), so we shall prove only (4.10) and (4.12).

Since $U_{n, 0}$ is nothing else but the $2 n$-th moment of the Wigner distribution on $(-2,2)$, we know, from the properties of the Wigner distribution, its generating function

$$
E_{0}(x):=\sum_{n=0}^{\infty} U_{n, 0} x^{n}
$$

is well defined on a neighborhood of the origin. So, Lemma (4.3) guarantees that for any $p \geq 0$, the generating function $E_{p}$ is well defined on a neighborhood of the origin.

In order to prove (4.12), we should calculate the generating function. By the definition and Lemma (4.2),

$$
E_{p}(z)=\sum_{n=0}^{\infty} U_{n, p} z^{n}=1+\sum_{n=1}^{\infty} \sum_{k=0}^{n-1} \frac{1}{1+p k} U_{k, p} U_{n-k-1, p} z^{n}
$$




$$
=1+z \cdot \sum_{k=0}^{\infty} U_{k, p} z^{k} \frac{1}{1+p k} \sum_{n=k+1}^{\infty} U_{n-k-1, p} z^{n-k-1} .
$$

By changing of variable $m:=n-k-1$ and rewriting the factor $\frac{1}{1+p k}$ as $\int_{0}^{1} y^{k p} d y,(4.14)$ is continuously equal to

$$
\begin{aligned}
& 1+z \cdot \sum_{k=0}^{\infty} U_{k, p} z^{k} \int_{0}^{1} y^{k p} d y \sum_{m=0}^{\infty} U_{m, p} z^{m} \\
& =1+z \cdot E_{p}(z) \sum_{k=0}^{\infty} U_{k, p} z^{k} \int_{0}^{1} y^{k p} d y \\
& =1+z \cdot E_{p}(z) \int_{0}^{1} \sum_{k=0}^{\infty} U_{k, p} z^{k} y^{k p} d y \\
& =1+z \cdot E_{p}(z) \int_{0}^{1} E_{p}\left(y^{p} z\right) d y .
\end{aligned}
$$

By the same arguments, we can study the characteristic function of the random variable $\left(A(1)+A^{+}(1)\right)$, say $F_{p}(t)$. In fact, Lemma (4.2) makes sure that the characteristic function $F_{p}$ has of form:

$$
F_{p}(t)=1+\sum_{n=1}^{\infty} \frac{U_{n, p}(i t)^{n}}{n !}
$$

By Lemma (4.1), $F_{p}$ is equal to

$$
\begin{aligned}
& 1+\sum_{n=1}^{\infty} \frac{(i t)^{n}}{n !} \sum_{k=0}^{n-1} U_{k, p} U_{n-1-k, p} \frac{1}{1+p k} \\
= & 1+\sum_{k=0}^{\infty} \frac{1}{1+p k} \sum_{n=k+1}^{\infty} \frac{(i t)^{n}}{n !} U_{k, p} U_{n-1-k, p} \\
= & 1+\sum_{k=0}^{\infty} U_{k, p} \frac{1}{1+p k} \sum_{m=0}^{\infty} \frac{(i t)^{m+k+1}}{(m+k+1) !} U_{m, p} \\
= & 1+i \sum_{k=0}^{\infty} U_{k, p} i^{k} \int_{0}^{1} y^{p} d y \int_{0}^{t} d t_{1} \int_{0}^{t_{1}} d t_{2} \cdots \int_{0}^{t_{n}} d t_{n+1} F_{p}\left(t_{n+1}\right) .
\end{aligned}
$$

It is important to give an explicit expression to the sequence $\left\{U_{n, p}\right\}_{n=0}^{\infty}$. For this, we have the following more general result:

Lemma (4.5). For each $n \in \mathbf{N}, \varepsilon \in\{0,1\}_{+}^{2 n}$,

$$
\left\langle\Phi, \prod_{h=1}^{2 n} A^{\varepsilon(h)}\left(g_{h}\right) \Phi\right\rangle=\prod_{h=1}^{n} \frac{1}{1+\frac{p}{2}\left(r_{h}-l_{h}-1\right)}\left\langle g_{l_{h}}, g_{r_{h}}\right\rangle .
$$


Proof. We prove the result by applying the induction. In the case of $n=1$, the thesis is obvious. Suppose that (4.17) is true for any $n \leq m$ and $\varepsilon \in\{0,1\}_{+}^{2 m}$, we shall prove that (4.17) is still valid for $n=m+1$.

Let be given an non-crossing pair partition $\varepsilon \in\{0,1\}_{+}^{2(m+1)}$ and denote by $\left\{l_{h}, r_{h}\right\}_{h=1}^{m+1}$ its left-right-index set. If the non-crossing pair partition $\varepsilon$ is not total connected, we consider its total-connected decomposition $\varepsilon \in\{0,1\}_{+}^{2 m_{i}}, i=1, \ldots$, $M \leq m$. Any $\varepsilon_{\imath}$ is total connected with the left-right-index set $\left\{l_{h}^{(i)}, r_{h}^{(i)}\right\}_{h=1}^{m_{i}}$ and any $m_{\imath}$ is less than or equal to $m$.

By Lemma (3.5) and the assumption of the induction, we know that the left hand side of (4.17) is equal to

$$
\prod_{i=1}^{M} \prod_{h=1}^{m_{i}} \frac{1}{1+\frac{p}{2}\left(r_{h}^{(i)}-l_{h}^{(i)}-1\right)}\left\langle g_{l_{h}^{(i)}}, g_{r_{h}^{(i)}}\right\rangle .
$$

By the definition of the total connected decomposition, any $\varepsilon_{i}$ is a shift of $\varepsilon$ restricting on a subset of $\{1,2, \ldots, 2 m+2\}$. More precisely,

$$
\begin{gathered}
\varepsilon_{1}=\left.\varepsilon\right|_{\left\{1,2, \ldots, 2 m_{1}\right\}}, \\
\varepsilon_{i}=\left.u_{-}^{2 \sum_{j=1}^{m i-1}} \varepsilon\right|_{\left\{2 \sum_{j=1}^{m_{j-1}}+1,2 \sum_{j=1}^{m_{1}-1}+2, \ldots, 2 \sum_{j=1}^{\left.m_{j}\right\}},\right.}, j=2,3, \ldots, M .
\end{gathered}
$$

For any $h=1,2, \ldots, m+1$, the left-right indices $\left\{l_{h}, r_{h}\right\}$ is surely a left-right indices $\left\{l_{h}^{(i)}, r_{h}^{(i)}\right\}$ of (exactly) one of $\varepsilon_{i}$ for a certain $i=1,2, \ldots, M$. Moreover, by the construction of the total connected decomposition, any pair $\left\{l_{h}^{(i)}\right.$, $\left.r_{h}^{(i)}\right\}$ is a shift of (exactly) one of pair $\left\{l_{h}, r_{h}\right\}$. So, the quantity (4.18) is nothing else but the right hand side of (4.17).

If $\varepsilon \in\{0,1\}_{+}^{2(m+1)}$ is total connected, the (4.17) is just a consequence of Lemma (4.1), the expression (4.8) and the assumption of the induction. Therefore, the proof is completed.

An easy consequence of Lemma (4.5) is that

Conclusion. For each $n \in \mathbf{N}$,

$$
\left\langle\Phi,\left(A(1)+A^{+}(1)\right)^{2 n} \Phi\right\rangle=\sum_{\varepsilon \in\{0,1\}_{+}^{2 n}} \prod_{h=1}^{n} \frac{1}{1+\frac{p}{2}\left(r_{h}-l_{h}-1\right)} .
$$

Now we can state a result on the construction of the density function of the sum of the creation and annihilation function with the text function 1 . 
THEOREm (4.6). The density function of the random variable $A(1)+$ $A^{+}(1)$ (under the vacuum state) is equal to

$$
D_{p}(x)=\int d \mathbf{P} \frac{1}{2 \pi} \chi_{(\xi, \xi)}(x) \cdot \frac{1}{\xi} \sqrt{4-\frac{x^{2}}{\xi^{2}}} .
$$

Where, $\xi$ is a random variable on a certain probability space $(\Omega, \mathscr{F}, \mathbf{P})$ with odd moments zero and $2 n$-th moment

$$
\frac{4 n}{\mid\{n-\text { c.p.p. } 2 n\} \mid \sum_{\varepsilon \in\{0,1\}_{+}^{2 n}} \Pi_{h=1}^{n} \frac{1}{1+\frac{p}{2}\left(r_{h}-l_{h}-1\right)} .}
$$

Proof. The proof follows immediately by verifying the moments.

Remark. The existence of the random variable $\xi$ will be given elsewhere.

We call the distribution given by the density function $D_{p}$ the deformed Wigner distribution since $D_{p}$ is a generalization the density function of the Wigner distribution on the interval $(-2,2)$, which is nothing else but the density function $D_{0}$.

The next example is: there exists a measurable space $(S, \mathscr{\ell}, \nu)$ and a measurable function $\lambda(s, x)$ on the product space $S \times M$, such that

i) $0<\lambda(s, x) \leq b<\infty$

ii) the $n$-th interacting function $\lambda_{n}$ has of form:

$$
\lambda_{n}\left(x_{1}, \ldots, x_{n}\right)=\int_{S} \nu(d s) \prod_{h=1}^{n} \lambda\left(s, x_{h}\right)
$$

For each fixed $s \in S$, we define a Hilbert space $\mathscr{H}_{s}$ by introducing on $\mathscr{H}=$ $L^{2}(M, d \mu)$ the scalar product

$$
\langle f, g\rangle_{s}:=\int \mu(d x) \lambda(s, x) \bar{f}(x) \cdot g(x) .
$$

By $\Gamma_{0}\left(\mathscr{H}_{s}\right)$, we denote the Free Fock space over the Hilbert space $\mathscr{H}_{s}$. Notice that for each fixed $s \in S$, the Free Fock space is practically the same as the usual Free Fock space. So the usual creation and annihilation operators (depend on $s \in$ $S$ ) can be introduced by the usual way and in the following, they will be denoted by $A_{s}(f), A_{s}^{+}(g)$ respectively. It is easy to verify that $\left\{\mathscr{H}_{s}\right\}_{s \in S}$ forms a measurable 
field (of Hilbert spaces) and the family $\left\{A_{s}, A_{s}^{+}\right\}_{s \in S}$ is a measurable field of operators. So we have right to consider the direct integral of the Hilbert spaces and the family of the creation and annihilation operators with respect to the given measure $\nu$.

On the set

(4.23) $\mathscr{K}:=\left\{\xi(\cdot): S \rightarrow \mathscr{H}:\{\xi(s)\}_{s \in S}\right.$ is measurable and $\left.\int_{S}\langle\xi(s), \xi(s)\rangle_{s} \nu(d s)<\infty\right\}$ we define the scalar product

$$
\langle\xi(\cdot) \mid \eta(\cdot)\rangle:=\int_{S}\langle\xi(s), \eta(s)\rangle_{s} \nu(d s)
$$

Thus, $\mathscr{K}=\int_{S}^{\oplus} \mathscr{H}_{s} \nu(d s)$ is nothing else but the direct integral of the Hilbert spaces $\left\{\mathscr{H}_{s}\right\}_{s \in S}$. Similarly, for any $n \in \mathbf{N}$,

$$
A^{+}\left(g_{1}\right) \cdots A^{+}\left(g_{n}\right) \Phi=\int_{S} \nu(d s) A_{s}^{+}\left(g_{1}\right) \cdots A_{s}^{+}\left(g_{n}\right) \Phi_{s}
$$

where, $\Phi_{s}$ is the vacuum vector of the Free Fock space $\Gamma_{0}\left(\mathscr{H}_{s}\right)$. Moreover, for any $\varepsilon \in\{0,1\}^{n}$,

$$
A^{\varepsilon(1)}\left(g_{1}\right) \cdots A^{\varepsilon(n)}\left(g_{n}\right) \Phi=\int_{S} \nu(d s) A_{s}^{\varepsilon(1)}\left(g_{1}\right) \cdots A_{s}^{\varepsilon(n)}\left(g_{n}\right) \Phi_{s} .
$$

So, for any $f \in L^{2}(M, d \mu)$,

$$
\left\langle\Phi,\left(A(f)+A^{+}(f)\right)^{n} \Phi\right\rangle=\int_{S} \nu(d s)\left\langle\Phi_{s},\left(A_{s}(f)+A_{s}^{+}(f)\right)^{n} \Phi_{s}\right\rangle
$$

and which is equal to

$$
\int_{S} \nu(d s)\|f\|_{s}^{2 N} \cdot \mid\{n \text {-c.p.p.2N\}| }
$$

if $n=2 N$ and zero if $n$ is odd. Where

$$
w_{s}(x)=\chi_{\left[-\|f\|_{s},\|f\|_{s}\right]} \frac{2\|f\|_{s}^{-1}}{\pi} \sqrt{1-\frac{x^{2}}{\|f\|_{s}^{2}}}
$$

is the density function of the Wigner distribution on $\left[-\|f\|_{s},\|f\|_{s}\right]$.

Finally, we consider an example in which $M=\mathbf{R}^{d}$ with $d \geq 1$ and the interaction functions have of form: 


$$
\lambda_{n}\left(x_{1}, \ldots, x_{n}\right)=e^{-c \sum_{n=1}^{n-1} \sum_{m=n+1}^{n}\left|x_{n} \cdot x_{m}\right|}, \quad \forall n=1,2, \ldots
$$

where, $c>0$ is a constant. In this case, for any $\varepsilon \in\{0,1\}_{+}^{2 N}$ and text functions $g_{1}$, $g_{2}, \ldots, g_{2 N}$, we have that

$$
\begin{gathered}
\left\langle\Phi, A^{\varepsilon(1)}\left(g_{1}\right) \cdots A^{\varepsilon(2 N)}\left(g_{2 N}\right) \Phi\right\rangle= \\
=\int \mu\left(d y_{1}\right) \cdots \mu\left(d y_{N}\right) \prod_{h=1}^{N}\left(\bar{g}_{l_{h}} \cdot g_{r_{h}}\right)\left(y_{h}\right) \cdot e^{-c \sum_{h=1}^{n-1} \sum_{m=h+1}^{n}\left|y_{h} \cdot y_{m}\right| \cdot x_{\left(r_{n}+1, r_{n}+2, \ldots\right)}\left(r_{m}\right)} .
\end{gathered}
$$

The formula shows that this example is in fact a natural analogue of the example obtained from the central limit of the time evolution of the quantum electro-magnetic field (see [1,2]) and its proof is similar as what did there. Moreover, the distribution of the field operator in this case is under investigation (see [5]).

At the end of the paper, we would like to make some discussions. It is well known that the Wigner distribution was found from the investigation on some phenomena in heavy particle physics. Wigner presented the Wigner distribution (or semi-circle distribution) as some type of limit distribution of the biggest eigenvalue of some random matrices (see [6,7]). 30 years after, Voiculescu found the same distribution by studying Free type non-commutative probability (see $[8,9]$ and the references within) and Kümmerer, Speicher set and then Fagnola developed the corresponding Free stochastic calculus theory (see $[10,11,12,13]$ and the references within). The interacting Free Fock space comes, originally, from the investigation of the central limit behaviour of the time evolution operator of the quantum electric-magnetic field (see [1,2]) and the corresponding quantum stochastic calculus theory is set in [4,14] (the reference [14] is devoted to study the Free quantum stochastic calculus theory on some Hilbert modules). We can ask ourselves: Is it possible to get some interesting constructions of the interacting Free Fock space in the ways similar as did by Wigner or Voiculescu? Such investigation now is being performed.

Remark. Recently, some interacting Free Fock spaces have been constructed by central limit theorem (see $[15,16,17])$ and the distribution of field operator on some interacting Free Fock spaces is also be investigated (see $[18,19,20]$ ).

\section{REFERENCES}

[1] L. Accardi, Y. G. Lu, Wiener noise versus Wigner noise in quantum electrodynamics, Q-P-R-T, V III (1993), 1-18. 
[2] L. Accardi, Y. G. Lu, Quantum electrodynamics and semi-circle noise, to appear in: International Journal of Nonlinear Physics.

[ 3 ] Y. G. Lu, Interacting Boson Fock space, submitted to Sci. Sin (A).

[4] Y. G. Lu, Quantum Stochastic Calculus on the Interacting Free Fock Space, submitted to J. Funct. Anal.

[5] Y. G. Lu, Some remarks on the deformed Wigner law, in preparation.

[6] E. Wigner, Characteristic vectors of bordered matrices with infinite dimension, Ann of Math., 62 (1955), 548-564.

[7] E. Wigner, On the distribution of the roots of certain symmetric matrices, Ann of Math., 67 (1958), 325-327.

[8] D. Voiculescu, Free noncommutative random variables, random matrices and the $I I_{1}$ factors of free groups, Q-P-R-T, VI (1991), 473-487.

[9] D. Voiculescu, Symmetries of some reduced free product $C^{*}$-algebras, Lect. Notes. Math., no. 1132 (1983), 556-588.

[10] B. Kümmerer, R. Speicher, Stochastic integration on the Curtz algebra $O_{\infty}$. J. Funct. Anal. 103, no. 2 (1992), 372-408.

[11] R. Speicher, A new example of "independence" and "white noise". Proba. Th. Rel. Fields. 84 (1990), 141-159.

[12] R. Speicher, Survey on the stochastic integration on the full Fock space. Q-P-R-T VI (1991), 421-436.

[13] F. Fagnola, On quantum stochastic integration with respect to "free" noises. Q-P-R-T VI (1991), 285-304.

[14] Y. G. Lu, A note on free stochastic calculus on Hilbert modules, submitted to: Random operators.

[15] M. DeGiosa, Y. G. Lu, The free creation and annihilation operators as the central limit of quantum Bernoulli process, submitted to Ran. Op. Sto. Eq.

[16] M. DeGiosa, Y. G. Lu, From quantum Bernoulli process to creation and annihilation operators on interacting $q$-Fock space, submitted to Nagoya Math. J.

[17] M. DeGiosa, Y. G. Lu, The passage from $2 \times 2$ matrices model to an interacting free Fock space, preprint.

[18] Y. G. Lu, An Interacting Free Fock Space and the reciprocal-Wigner Law, to appear in Proba. Th. Math. Stat. (1997).

[19] Y. G. Lu, On the Anderson Type Interacting Free Fock Space, submitted to Ann. Proba.

[20] Y. G. Lu, S. Ruggeri, A new example of interacting Fock space and the distribution of field operator, submitted to Bollettino U. M. I.

Universitá degli studi di Bari

Dipartimento di Mate.

Campus Universitario

Via E. Orabona 4

70125 Bari, Italy 